\title{
Perfusion Computed Tomography for the Assessment of Myocardial Viability - a Case Series
}

\author{
Mirabela Morariu', Diana Opincariu², Alexandra Stănescu² \\ ${ }^{1}$ Clinic of Cardiology, University of Medicine and Pharmacy, Tîrgu Mureș, Romania \\ 2 Center of Advanced Research in Multimodality Cardiac Imaging, Cardio Med Medical Center, Tîrgu Mureș, Romania
}

\section{CORRESPONDENCE}

Diana Opincariu

76, 22 Decembrie 1989 St

540124 Tîrgu Mureș, Romania

Tel: 0265217333

Email: diana.opincariu@yahoo.ro

\section{ARTICLE HISTORY}

Received: 5 May, 2016

Accepted: 20 May, 2016 Email: mirabela.morariu@yahoo.com

Alexandra Stănescu • 76, 22 Decembrie 1989 St, 540124 Tîrgu Mures, Romania, Tel: +40 265217333 Email: alexandrastanescu90@gmail.com

\begin{abstract}
Myocardial viability plays an important role in preventing the development of left ventricular remodeling following an acute myocardial infarction. A preserved viability in the infarcted area has been demonstrated to be associated with a lower amplitude of the remodeling process, while the extent of the non-viable myocardium is directly correlated with the amplitude of the remodeling process. A number of methods are currently in use for the quantification of the viable myocardium, and some of them are based on the estimation of myocardial perfusion during pharmacologic stress. 64-slice Multi-detector Computed Tomography (MDCT) during vasodilator stress test, associated with CT Coronary Angiography (CCTA) has a high diagnostic accuracy in evaluating myocardial perfusion. In this article, we present a sequence of 3 clinical cases that presented with symptoms of myocardial ischemia, who underwent 64-slice MDCT imaging at rest and during adenosine stress test, in order to assess the extent of the hypoperfused myocardial areas. Coronary artery anatomy and the Coronary Calcium Score was assessed for all 3 patients by performing CT Coronary Angiography. The combination of CT Angiography and adenosine stress CT myocardial perfusion imaging can accurately detect atherosclerosic lesions that cause perfusion abnormalities, compared with the combination of invasive angiography and single-photon emission computed tomography (SPECT).
\end{abstract}

Keywords: perfusion computed tomography, myocardial viability, ventricular remodeling

\section{INTRODUCTION}

Left ventricular remodeling after an acute myocardial infarction is one of the most powerful predictors of long-term patient evolution. In this process of remodeling, an important role is played by myocardial viability, which has been demonstrated to be associated not only with a lower amplitude of the remodeling process, but also with a major regression following revascularization. ${ }^{1}$

After an acute myocardial infarction, the identification of stunned myocardium has a major importance, because it represents the area of viable myocardium that can recover its function if a therapeutic intervention is done in order to improve myocardial perfusion, mainly consisting of interventional revascularization. A ne- 
crotic myocardium, where there is no evidence of viability, is an inert tissue and it does not respond well to therapeutic measurements. The extent of the viable myocardium at the periphery of the infarcted area is correlated directly and negatively with the amplitude of the remodeling process. ${ }^{2,3}$

A number of methods are proposed for the quantification of the viable myocardium, in order to establish adequate treatment. 64-slice Multi-detector Computed Tomography (MDCT) during vasodilator stress test, combined with CT Coronary Angiography (CCTA) has a high diagnostic accuracy in evaluating myocardial perfusion. ${ }^{4-7}$

The primary aim of the perfusion CT is to detect myocardial ischemia and to identify the patients that would benefit most - patients with intermediate to high pre-test probability of coronary artery disease (CAD), patients with high Calcium Scores, patients with known CAD, and those with previous myocardial infarction. 8,9
Other methods with high sensibility and specificity that are used for the evaluation of myocardial viability are lowdose dobutamine stress echocardiography, single photon emission tomography (SPECT), positron emission tomograph (PET), magnetic resonance imaging (MRI).10-12

\section{CASE SERIES}

In this article, we present a sequence of 3 clinical cases that presented with symptoms of myocardial ischemia, who underwent 64-slice MDCT imaging, at rest and during adenosine stress test, in order to assess the extent of the hypoperfused areas. The coronary artery anatomy and the Coronary Calcium Score was assessed for all 3 patients, by performing CT coronary angiography.

The pharmacological stress test was induced by administering adenosine, a vasodilator agent, which was injected
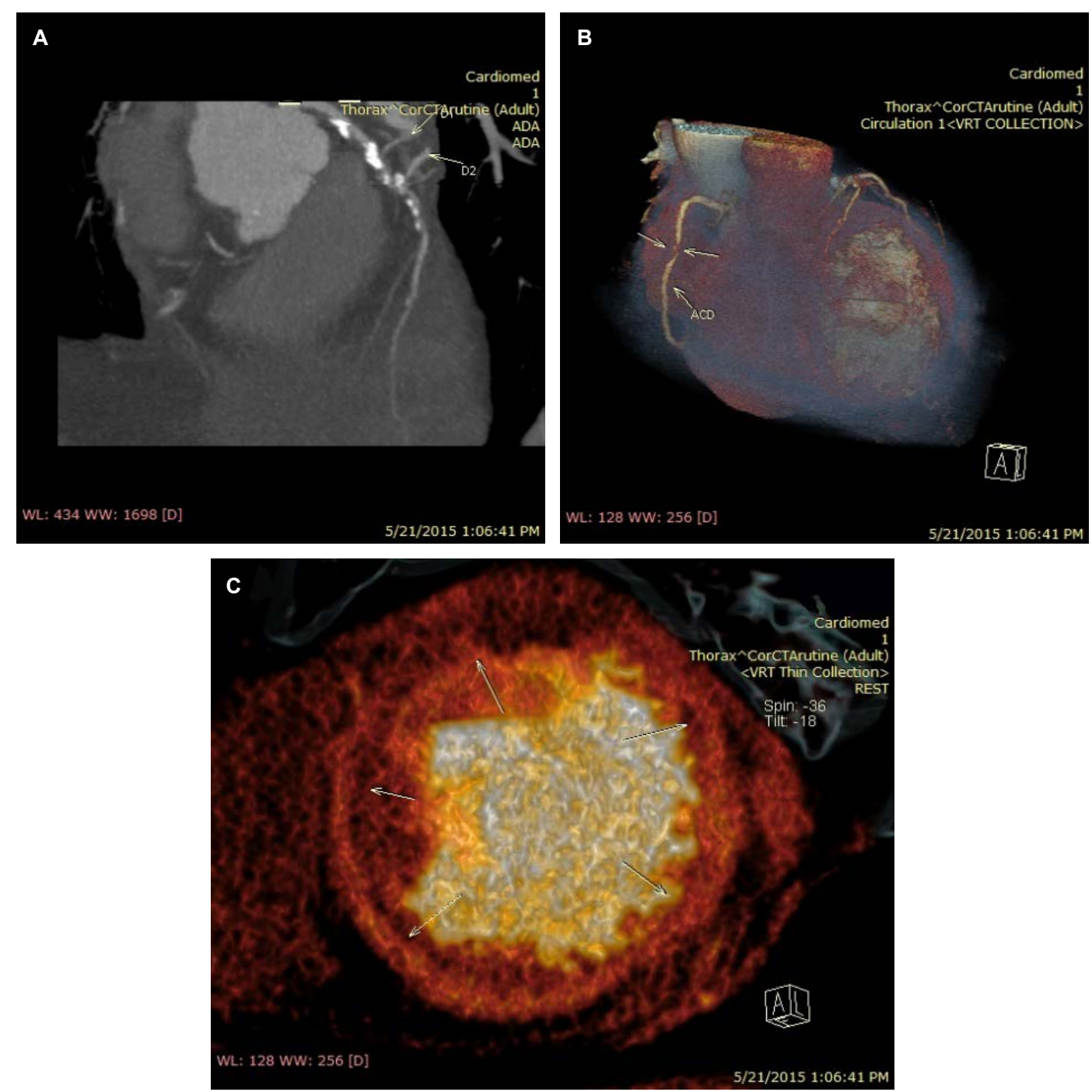

FIGURE 1. A - High calcium content in the LAD; $\mathbf{B}$ - Severe stenosis in the RCA; C - Partially reversible hypoperfusion in the apical area 

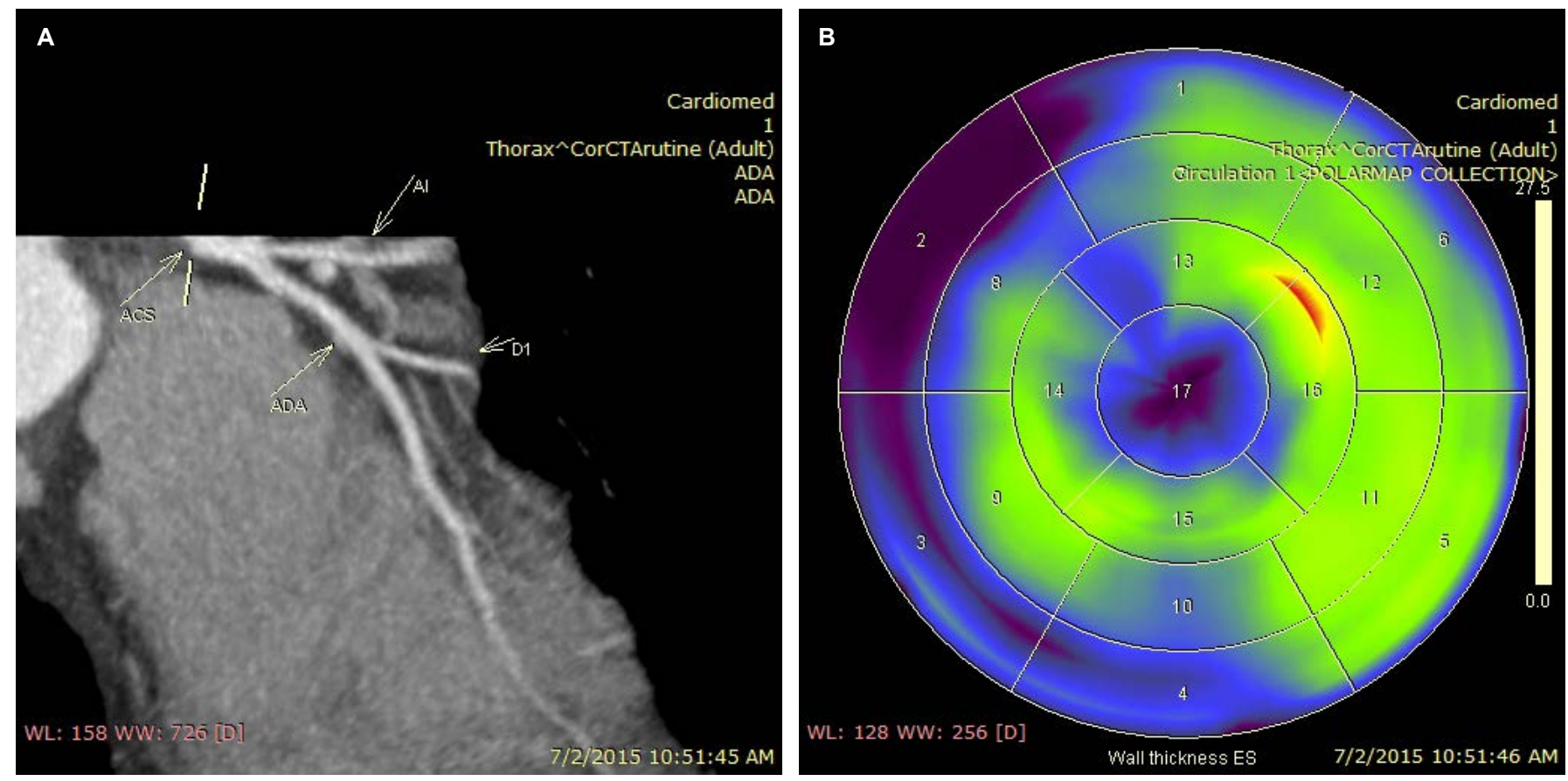

FIGURE 2. A - Intramuscular trajectory of the left anterior descending artery in the distal segment; B - Hypodense lesion in the subendocardium, that was extended to the anterolateral wall of the LV.

over a minimum duration of $2-3$ minutes with concomitant electrocardiographical monitoring. The rest aquisition was performed after a sufficient time (15-20 minutes) in which the patients were in a relaxed state.
The 64-slice MDCT examination, focused on the coronary arteries, was performed by dynamic administration of $100 \mathrm{ml}$ non-ionic contrast agent (lopamiro 370) followed by $100 \mathrm{ml}$ saline wash, by an antecubital venous line.
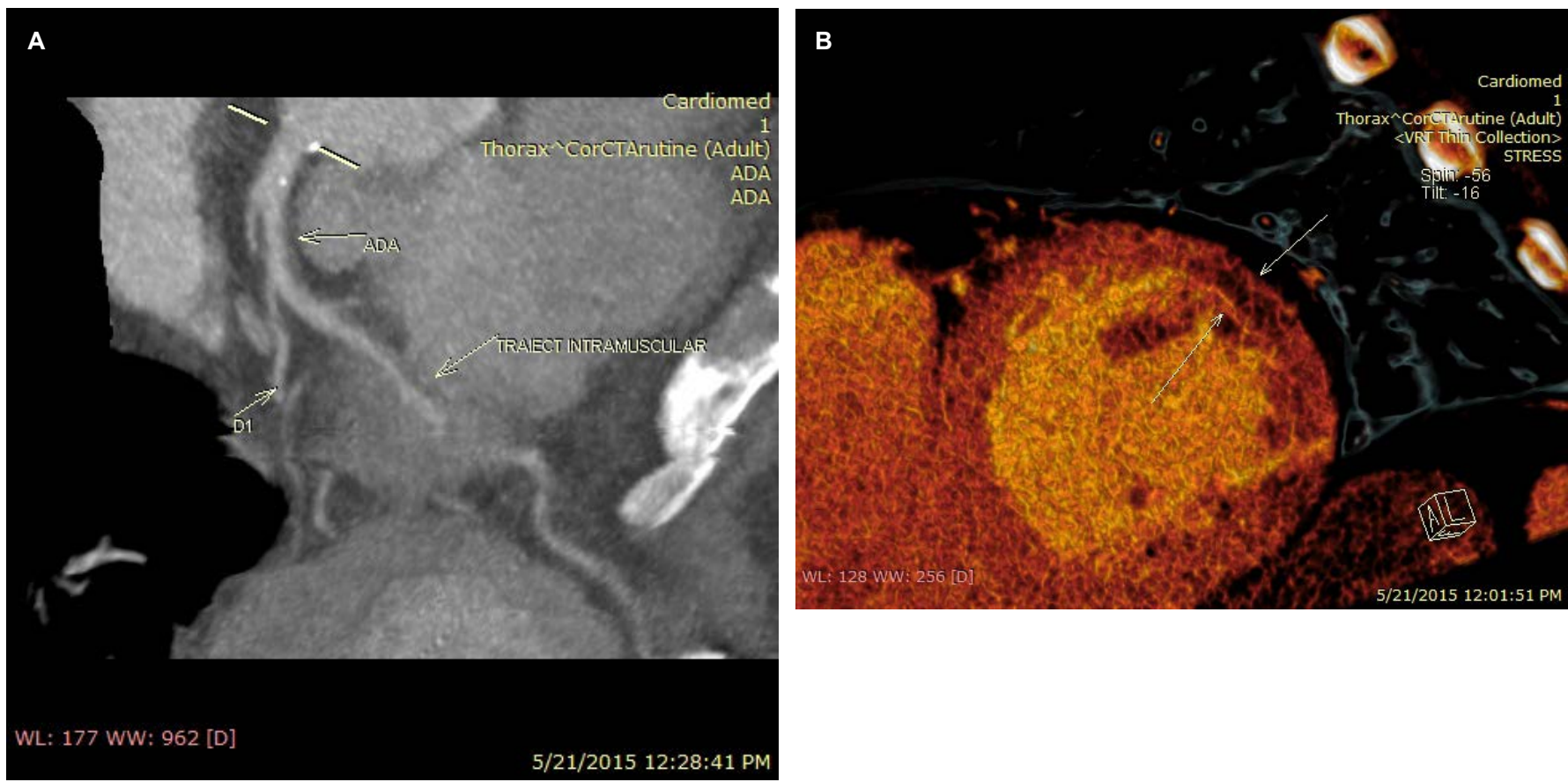

FIGURE 3. A - Intramuscular pathway of the LAD at the level of the middle segment; $\mathbf{B}$ - Subendocardial hypodense lesion in the anterolateral wall of the LV in the middle segment 
The examination was well tolerated, without any complications.

The CT examination protocol that was used consisted in the positioning of an intravenous catheter, the continuous monitoring of the 12-lead ECG and blood pressure, and also a close monitorization of symptoms and vital signs.

The pharmacological stress test with adenosine induces a coronary steal phenomenon (the normal coronary arteries dilate more than the arteries affected by atherosclerosis), leading to a difference in flow, therefore identifying the underlying perfusion defect in the territory supplied by the diseased coronary artery. Adenosine acts directly on adenosine receptors, leading to coronary vasodilation; it has a rapid onset and a short duration of action..$^{13,14}$ Thus, a continuous infusion is required for the myocardial CT stress perfusion test (CTP), which possesses a good sensitivity and specificity in detecting myocardial perfusion defects. Side effects include reflex tachycardia, and it is contraindicated in patients with asthma, chronic obstructive pulmonary disease and atrioventricular block.

All patients consented to publication of their data and the publication was approved by the Ethics Committee of the center where the examinations were performed. All the examinations were performed in accordance to the principles stated in the Declaration of Helsinki.

The initial results using 64-MDCT have been promising, showing that myocardial CT perfusion protocol has comparable diagnostic characteristics to SPECT MPI in the detection of myocardial perfusion defects. ${ }^{15}$

The myocardial CT perfusion protocol allows simultaneous acquisition of coronary anatomy imaging and myocardial perfusion, and a combined CTA/CTP protocol has been shown to have better diagnostic characteristics than CTA alone.

The radiation exposure in such protocols is similar to that of a traditional SPECT MPI examination. These data suggest that myocardial CTP has the potential to become a robust clinical tool for the evaluation of chest pain patients. ${ }^{16}$

The first case is a male patient known with diabetes mellitus, who presented with an acute myocardial infarction at 18 hours from the onset of symptoms. The Coronary Calcium Score was assessed at 645, showing a very high cardiovascular risk (Figure 1, Diagram A). The coronary anatomy imaging described a $40 \%$ aorto-ostial stenosis in the Left Main Artery, moderate and severe stenosis in the Left Anterior Descending Artery (LAD) and a severe lesion in the vertical segment of Right Coronary Artery (RCA) (Figure 1, Diagram B). Furthermore, he had an associated left ventricular (LV) aneurism in the apical segment.
The rest phase $\mathrm{CT}$ acquisition detected a hypodense lesion in the subendocardium at the apex of the LV, that was shown to be significantly lower during the stress phase acquisition. This lesion was interpreted as a partially reversible hypoperfusion, possibly due to the vasodilator effect of adenosine, which was used as a myocardial stress agent (Figure 1, Diagram C).

The second case is a male patient who presented with atypical angina pectoris. The Coronary Calcium Score was calculated at 0 , therefore indicating a low cardiovascular risk.

The coronary anatomy assessment showed an intramuscular trajectory of the Left Anterior Descending Artery in the distal segment (length of $22 \mathrm{~mm}$ ) and Intermediate Artery in the distal segment, without any other significant atherosclerotic lesions (Figure 2, Diagram A). The rest phase CT acquisition detected a hypodense lesion in the subendocardium, that was extended to the anterolateral wall of the LV. The hypodensity was absent during the stress phase acquisition, thus indicating that the perfusion defect is reversible (Figure 2, Diagram B).

The third case is a male patient with known arterial hypertension, permanent atrial fibrillation, a positive history of stroke, who presented with an anterior myocardial infarction at more than 48 hours from the onset of symptoms. The coronary anatomy evaluation showed an intramuscular pathway of the LAD at the level of the middle segment, without any significant atherosclerotic lesions (Figure 3, Diagram A). The CT stress acquisition revealed a subendocardial hypodense lesion in the anterolateral wall of the LV in the middle segment, which had been absent during the rest acquisition (Figure 3, Diagram B).

\section{DISCUSSIONS}

Computed Tomography is the most frequent investigation used for the evaluation of coronary anatomy and it has been used as a risk stratification tool for the exclusion of CAD. Myocardial stunning occurs in patients with coronary artery disease in situations in which the myocardium is exposed to transient ischemia, such as unstable angina, exercise-induced ischemia, acute myocardial infarction with early reperfusion, open heart surgery, and cardiac transplantation. ${ }^{17}$

The combination of CT angiography and adenosine stress CT myocardial perfusion imaging can accurately detect atherosclerotic lesions that cause perfusion abnormalities, compared with the combination of invasive angiography and single-photon emission computed tomography (SPECT). ${ }^{10,11,15}$ 
Areas with constant hypoattenuation during both phases correspond to irreversible ischaemia. Reversible ischemia can be suspected if hypoattenuation is present only in the stress phase or if the hypoattenuated area increases significantly. ${ }^{18}$

In the first case, the presence of ischemia in the anterior wall of the LV was correlated with the presence of a perfusion deficit which was partially reversible during stress acquisition.

In the second and the third cases, the presence of an intramuscular trajectory of the Left Anterior Descending Artery correlated with a reversible perfusion deficit in the antero-lateral wall of the LV.

CT perfusion imaging, when performed with adenosine stress test, can detect subendocardial perfusion deficits. The combination of CT angiography and CT perfusion imaging can detect obstructive atherosclerosis that causes perfusion abnormalities, highly comparable with the combined gold standard of invasive angiography and singlephoton emission computed tomography myocardial perfusion imaging.

\section{CONFLICT OF INTEREST}

Nothing to disclose.

\section{ACKNOWLEDGEMENT}

This research was supported by the Cardio Med Medical Center, project no. CAM/2015/RD/41.

\section{REFERENCES}

1. van Loon RB, Veen G, Kamp O, Baur LHB, van Rossum AC. Left ventricular remodeling after acute myocardial infarction: the influence of viability and revascularization - an echocardiographic substudy of the VIAMI-trial. Trials. 2014;15:329.

2. Elfigih IA, Henein MY. Non-invasive imaging in detecting myocardia viability: Myocardial function versus perfusion. Int J Cardiol Heart Vasc. 2014;5:51-56

3. Shaw LJ, Berman DS, Maron DJ, et al. Optimal medical therapy with or without percutaneous coronary intervention to reduce ischemic burden: results from the Clinical Outcomes Utilizing Revascularization and Aggressive Drug Evaluation (COURAGE) trial nuclear substudy. Circulation. 2008:117:1283-1291.

4. Cury RC, Nieman K, Shapiro MD, et al. Comprehensive assessment of myocardial perfusion defects, regional wall motion, and left ventricular function by using 64-section multidetector CT. Radiology. 2008;248:466475.

5. Meijboom WB, Meijs MF, Schuijf JD, et al. Diagnostic accuracy of 64-slice computed tomography coronary angiography: a prospective, multicenter multivendor study. J Am Coll Cardiol. 2008;52(25):2135-2144.

6. Di carli MF, Dorbala S, Curillova Z, et al. Relationship between CT coronary angiography and stress perfusion imaging in patients with suspected ischemic heart disease assessed by integrated PET-CT imaging. J NuCl Cardiol. 2007;14:799-809.

7. Blankstein R, Shturman LD, Rogers IS, et al. Adenosine-induced stress myocardial perfusion imaging using dual-source cardiac computed tomography. J Am Coll Cardiol. 2009;54:1072-1084.

8. Budoff MJ, Gul KM. Expert review on coronary calcium. Vasc Health Risk Manag. 2008;4(2):315-324

9. Greenland P, Bonow RO, Brundage BH, et al. ACCF/AHA 2007 clinical expert consensus document on coronary artery calcium scoring by computed tomography in global cardiovascular risk assessment and in evaluation of patients with chest pain: a report of the American College of Cardiology Foundation Clinical Expert Consensus Task Force (ACCF/AHA Writing Committee to Update the 2000 Expert Consensus Document on Electron Beam Computed Tomography) developed in collaboration with the Society of Atherosclerosis Imaging and Prevention and the Society of Cardiovascular Computed Tomography. J Am Coll Cardiol. 2007;49(3):378-402

10. Partington SL, Kwong RY, Dorbala S. Multimodality imaging in the assessment of myocardial viability. Heart Fail Rev. 2011;16(4):381-395.

11. Hachamovitch R, Hayes SW, Friedman JD, Cohen I, Berman DS. Comparison of the Short-Term Survival Benefit Associated With Revascularization Compared With Medical Therapy in Patients With No Prior Coronary Artery Disease Undergoing Stress Myocardial Perfusion Single Photon Emission Computed Tomography. Circulation. 2003;107:2900-2907.

12. Gaemperli O, Husmann L, Schepis T, et al. Coronary CT angiography and myocardial perfusion imaging to detect flow-limiting stenoses: a potential gatekeeper for coronary revascularization? Eur Heart J. 2009;30:29212929

13. Chen JF, Eltschig HK, Fredholm BB, et al. Adenosine receptors as drug targets - What are the challenges? Nat Rev Drug Discov. 2013;12(4):26586.

14. Levine MG, Ahlberg AW, Mann A, et al. Comparison of exercise, dipyridamole, adenosine, and dobutamine stress with the use of Tc-99m tetrofosmin tomographic imaging. J Nucl Cardiol. 1999;6:389-396.

15. Cheng W, Zeng M, Arellano C, et al. Detection of myocardial perfusion abnormalities: standard dual-source coronary computed tomography angiography versus rest/stress technetium-99m single-photo emission CT. Br J Radiol. 2010;83:652-660.

16. Techasith T, Cury RC. Stress Myocardial CT Perfusion: An update an future perspective. J Am Coll Cardiol. 2011;8(4):905-916.

17. Kloner RA, Bolli R, Marban E, Reinlib L, Braunwald E. Medical an Cellular Implications of Stunning, Hibernation, and Preconditioning - An NHLBI Workshop. Circulation. 1998;97:1848-1867.

18. Bucher AM, De Cecco CN, Schoepf UJ, et al. Cardiac CT for myocardial ischaemia detection and characterization - comparative analysis. $\mathrm{Br} J$ Radiol. 2014;87:20140159. 